\title{
Synchronizing Chaotic Optically-Programmable Digital Circuits
}

\author{
J.A. Martín-Pereda, A. González-Marcos and C. Sánchez-Guillén \\ E.T.S. Ingenieros de Telecomunicación \\ Universidad Politécnica de Madrid \\ 28040 Madrid. Spain \\ $+34.1 .336 .7304$ \\ jamp@atfo.upm.es
}

\begin{abstract}
In this paper an approach to the synchronization of chaotic circuits has been reported. It is based on an optically programmable logic cell and the signals involved are fully digital. It is based on the reception of the same input signal on sender and receiver and from this approach, with a posterior correlation between both outputs, an identical chaotic output is obtained in both systems. No conversion from analog to digital signals is needed. The model here presented is based on a computer simulation.
\end{abstract}

\section{1.- Introduction}

In the last years there has been a considerably interest in utilizing chaotic circuits to implement secure communication systems. One of the main problems to be attended is the one concerning synchronized circuits. The idea that chaotic systems could synchronize was first put forth in a paper almost ten years ago [1]. Recently, Pecora and Carroll, [2]-[4] have shown that is possible to construct a set of chaotic systems such that their common signals are synchronized. Several authors have followed the same concepts and a large number of papers have been focused on such a topic. Moreover, some schemes using the Chua's circuit have appeared in the literature following similar ideas [5].

A new scheme is proposed in this paper. The basis is the use of an Optically-Programmable Digital Circuit, reported previously by us [6]-[10], and able to process two input binary signals. Its two outputs are logical functions of these inputs. The type of processing is related to the eight main Boolean Functions, namely, AND, OR, XOR, NAND, NOR, XNOR, ON and OFF. The programmable ability of the two outputs, as it has been described, allows the generation of several datacoding for optical transmission. Moreover, as it was shown, this circuit has the possibility to.the generation of periodic and even chaotic solutions. A precise analyze of the output characteristic, versus the main variable parameters, as control signal level and data signal level, has given some results which were described with the help of the Chaos Theory [10].

Another important fact to be taken into consideration is the digital character of the obtained signal. While most of the chaotic results reported in the literature are analog and they need to be converted to digital if such a signal is needed, in our case a digital chaos is obtained directly from the structure.

\section{2.- Cell description}

Although a complete description of the basic blocks and behaviour of our structure has been given in some previous papers [6],[11], the main concepts will be reviewed here.

A block diagram of the circuit is show in Fig. 1. As it can be seen, the circuit is composed by two devices, $\mathrm{P}$ and $\mathrm{Q}$, with a non-linear behaviour. The outputs of each one of them correspond to the two final outputs, $\mathrm{O}_{1}$ and $\mathrm{O}_{2}$, of the cell. The possible inputs to the circuit are four. Two of them are for input data, $\mathbf{I}_{1}$ e $\mathbf{I}_{2}$, and the other two, $\boldsymbol{g}$ and $\boldsymbol{h}$, for control signals. The way these four inputs are arranged inside the circuit, is also

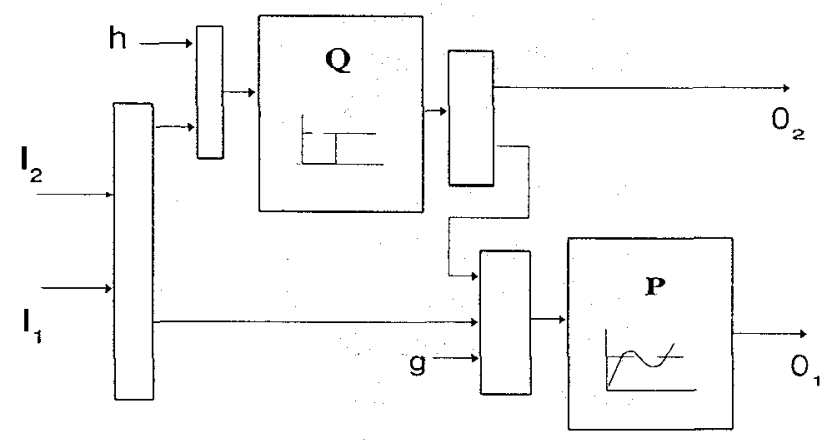

Figure 1 Basic structure of the Optical Programmable Logic Cell. 
represented in Figure 1. The corresponding inputs to the non-linear devices, $\mathrm{P}$ and $\mathrm{Q}$, are functions of these signals plus, in the case of the $\mathrm{P}$ device, one other coming from inside the own cell and obtained from the $\mathrm{Q}$ device.

A practical implementation we have carried out

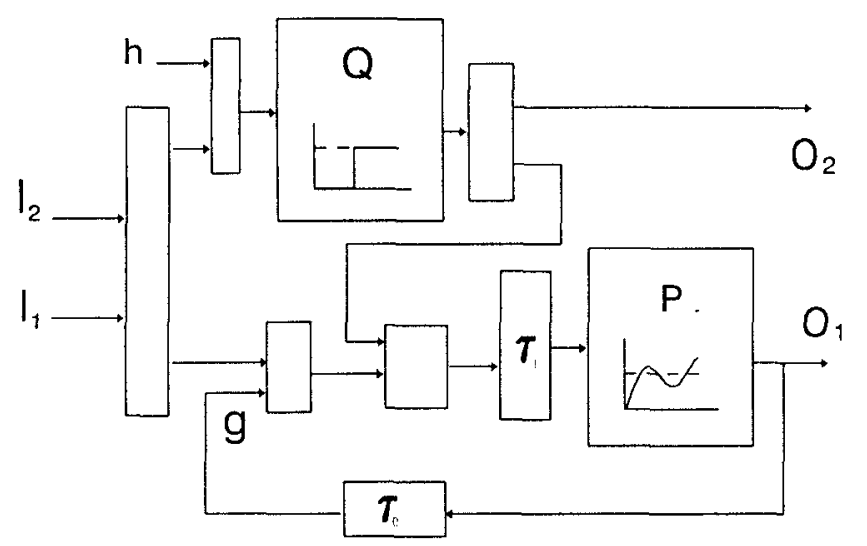

Figure 2 Configuration of the OPLC with feedback.

of the processing element has been based on an optoelectronical configuration. Lines in Fig. 1 represent optical multimode fibers. The indicated blocks, placed in order to combine the corresponding signals, are conventional optical couplers. In this way, optical inputs arriving to the individual devices are multilevel signals.

The characteristics of the non-linear devices are also shown in Fig. 1. Device Q, corresponds to a thresholding or switching device, and device $\mathrm{P}$ is a multistate device, being the response of this non-linear optical device the one represented in Fig. 1. This response is similar to the one had by a SEED device. Because the input signal is a multilevel signal the output depends on the relations between:

a) the level of a bit "1",

b) the level of the control signal, and

c) the level for switching from one state to another. This level is intrinsic to the employed device.

In the results to be reported here, we have not paid too much attention to the experimental implementation but to its computer simulation. It has been done with the MATLAB ${ }^{\mathrm{TM}}$ program and the SIMULINK $^{\mathrm{TM}}$ application.

The value of a bit "1" at any of the two inputs of the cell, namely $I_{1}$ or $I_{2}$, has been considered as normalization value for the present simulation of the Optical-Programmable Logic Circuit.

Another way to work with this cell is the possibility to control the decision levels. These levels are

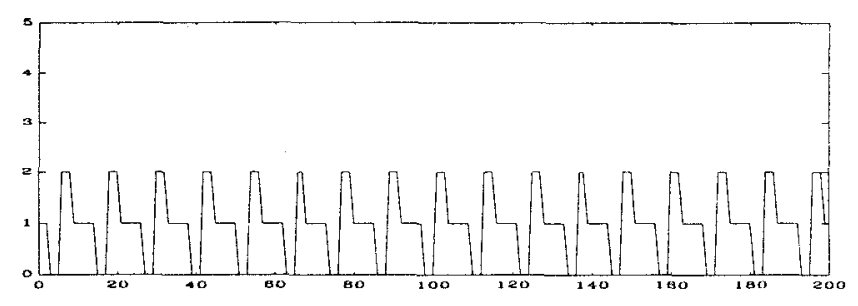

Figure 3 Input to the P device before feedback.

intrinsic to each particular employed device. In our case, and by maintaining the same normalization, different logic tables are possible to be obtained by changing these decision level values. As it was shown [10], a large variety of logic functions are obtained in a very simple way. Moreover, a particular point needs to be pointed out. It concerns the extreme sensitivity of the output logic functions with respect to both control signals and decision levels. This fact is of a great importance for further considerations on the working conditions.

\section{3.- Periodic and Chaotic Behaviour}

A non-linear behaviour can be expected from the above reported cell, if some kind of feedback should be applied. Some examples of this type of behaviour are well-known from the literature [11]-[13]. Although the present configuration has almost no points in common

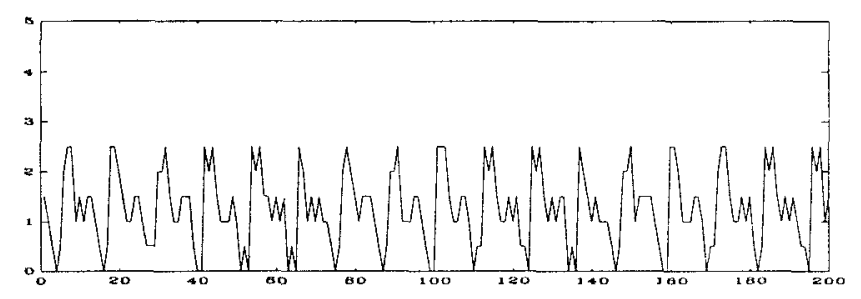

Figure 4 Input to the $P$ device: feedback plus input signals.

with the cases reported, the presence of non-linear devices in the system gives some analogies with them. 
In order to study the non-linear response of our circuit, some minor modifications were performed. The first one was to introduce a feedback from one of the two possible outputs to one or both of the cell inputs. Moreover, according to previous studies in this field, the introduced feedback has to have some time delay. In the same way, because the results we are going to get will be obtained by computer simulation, another internal delay was needed. It corresponds to the real response time of the simulated nonlinear devices, $\mathbf{P}$ and $\mathbf{Q}$.

In general, a periodic behaviour should be expected as the normal output of the system. But, under some conditions, this is not always true: the output is not periodic with some parameters values of the system.

The feedback applied to the system, among the different possibilities, is the one corresponding from the output $\mathrm{O}_{1}$ of Q-device (see Fig. 1) to the control input, $g$, of P-device. No other additional control signal has been used. Fig. 2 shows the final circuit with feedback.

According to previous studies [11]-[13], the situation with more probability to give a periodic or even chaotic solution is when internal delay time is shorter than the external one. In everyone of our studied cases, a regular train of pulses has been the input. The real input to the device $\mathrm{P}$, before the feedback takes place, is shown in Fig. 3. As it can be seen, it is a multilevel signal corresponding to the addition of the two inputs. The period of this signal, in this particular case, corresponds to a time of 14 bits.

If the ratio between internal and external delay times is smaller than 1 , we obtain a periodic situation. Some examples are given in references [9]-[10]. But a chaotic output is obtained when the internal response time equal to zero or much smaller than the external one. Input signal, corresponding to feedback plus input data, to P-device control gate, is shown in Fig. 4. Some output signals are given in [6]. No indication of a possible periodic behaviour has been obtained and, as it has been shown, it shows chaotic properties. They have been studied by the Lempel and Ziv [14] and the autocorrelation [15] methods.

\section{Synchronizing Optical Programmable Logic Cells}

If two identical cells, as the above mentioned, with feedback, are parallel connected and the same signals arrives to their inputs, an identical chaos is obtained at their outputs. The situation is shown in Fig. 5. This situation has been easily obtained by computer simulations. No particular problems are present when a configuration as the one here reported is employed. This situation corresponds to two identical and ideal configurations working under identical conditions. A similar method to the previously reported by us to study

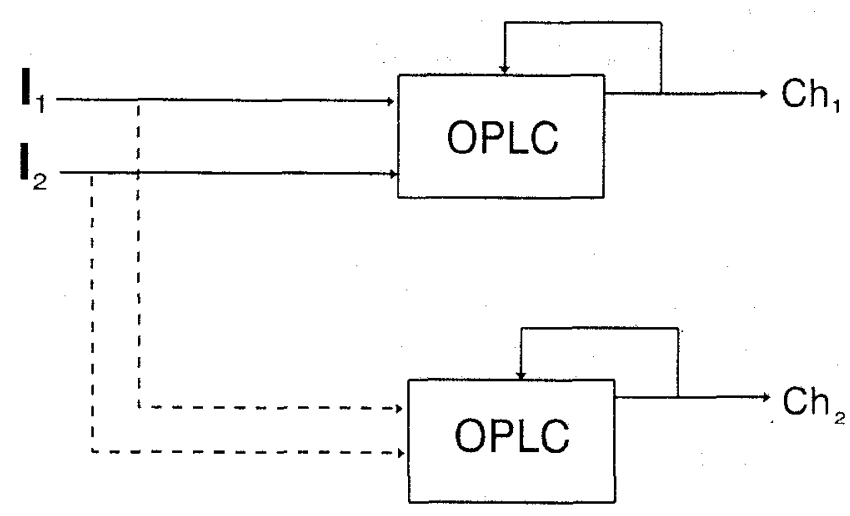

Figure 5 Chaotic circuits with identical inputs and outputs.

chaos [10] was employed in order to compare both output signals. They were converted to hexadecimal and represented in an $\mathrm{x}-\mathrm{y}$ graph. The $\mathrm{x}$-axis corresponds to the output signal from one of the OPLCs and the $y$-axis to the other one. An straight line was obtained.

Because this is not a real situation some modifications have to be adopted.

The behaviour becomes more critical when the simulation tries to be close to a real situation. In this case, if both systems are not feed by exactly the same signal, the obtained outputs, although chaotics, are different. Hence, no possible relation between then should be feasible.

The solution we have adopted is presented in Fig. 6. Both systems, drive and response, are located at different places. As a consequence, there is no possibility to introduce the same input signals to their corresponding input ports simultaneously. But because just one of these two input signals is needed to generate both, this signal is the one sent to both structures. The second one, if needed, may be locally generated by the corresponding circuit, either optical or electronic depending on the type of system. A variable time delay is added to the first system in order to obtain the same retard at the entrance of its OPLC. We will show later the importance of this time delay.

The chaotic output from the first system is added to the information signal. The new one is then transmitted and compared at the receiver with the obtained chaos from the second OPLC. If both systems are perfectly synchronized, it will be possible to obtain the information signal, by subtracting signal plus chaos 
minus chaos.

The computer simulation we have adopted, shown in Fig. 6, takes into account the above mentioned facts. As it can be seen, one single signal, I, is received by both systems. It becomes the two required logic data sent to the receiver.

Some considerations are needed to take into account with respect to the initial delay time at the OPLC1 input. In a practical situation, no precise idea is had about its exact value, knowing only the order of

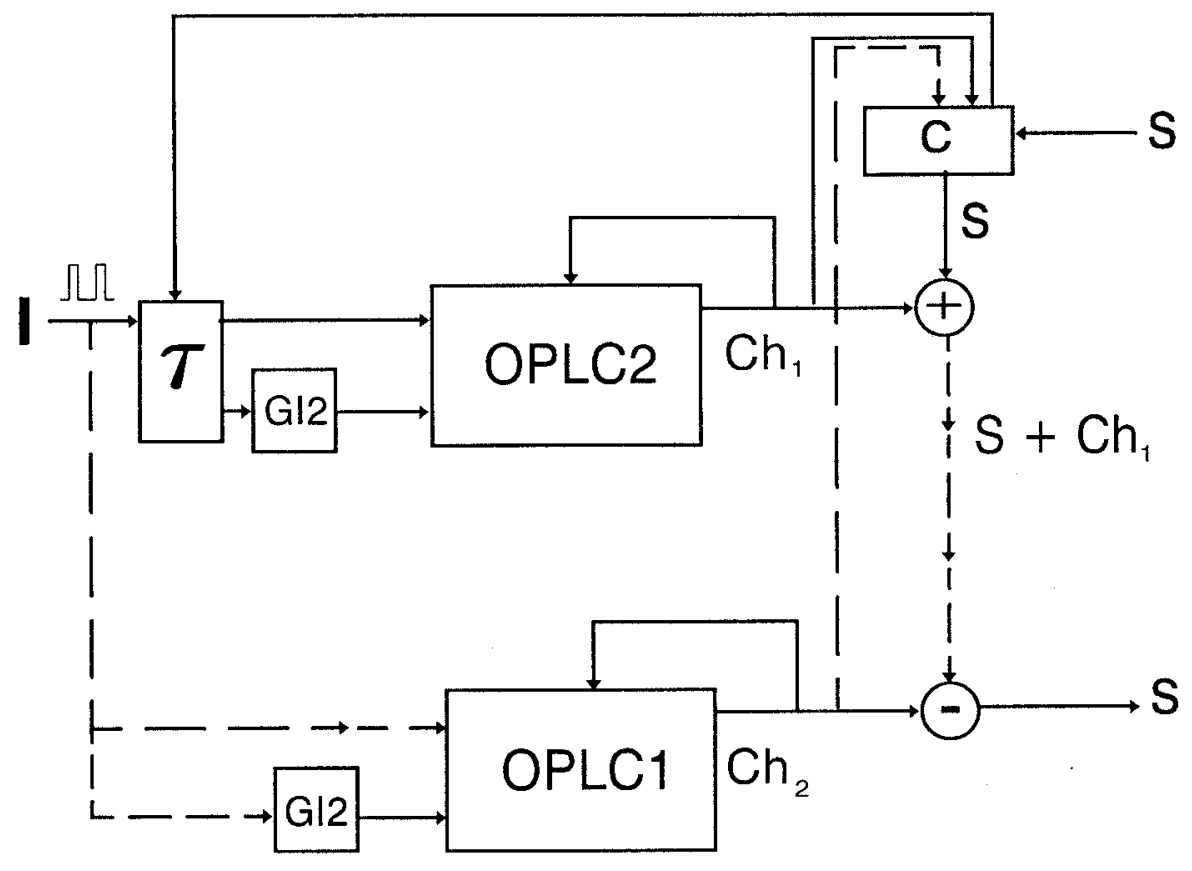

Figure 6 General configuration of the chaotic synchronized circuits.

for the OPLCs. With these trains of pulses, OPLCl and OPLC become chaotic. We have to remember that although both logic cells are represented at Fig. 6 with different names, they have to be identical. This is a necessary condition.

Another point needs to be considered. Two blocks, named as GI2 at Fig. 6, appear before the logic cells. Both circuits are identical in receiver and sender and are able to generate the second input signals needed. Their characteristics can be varied according to a previous established code adding greater freedom and security.

A further provision has to be implemented in order to synchronize both chaos. We have obtained it by comparison at $\mathrm{C}$. The second system send its output to $\mathrm{C}$ where is compared with the one obtained from OPLC1.

Different are the possible ways to perform the above mentioned comparison. In the present case we have just made a direct substraction of both signals and assumed both chaos are identical if after a certain number of time intervals the result is zero. At this moment, signal is added to the signal from OPLC2 and magnitude. Hence, the time delay has to be varied around a certain range. These variations are imposed by the signal obtained at $\mathrm{C}$. A method to evaluate this range can be the same hexadecimal method explained before [10]. This signal is feedbacked to the time delay generator $\tau$ that controls the time delay to the OPLC2 cell.

\section{5.- Conclusions}

A different approach to the synchronization of chaotic circuits has been reported. As based on an optically programmable logic cell its output is digital. Hence, the application to optical communications comes directly from its properties. The model here presented is based on a computer simulation. A laboratory implementation will be needed to confirm our approach.

\section{Acknowledgments}

This work was partly supported by the Comisión Interministerial de Ciencia y Tecnología, CICYT, grant TIC95-0118. 


\section{References}

[1] V.S. Afraimovich, N.N. Verichev and MI. Rabinovich, "Stochastic synchronization of oscillations in dissipative sytems", Inv. VUZ. Rasiofiz. RPQAEC 29, 795-803, 1986.

[2] L.M. Pecora and T.L. Carroll, "Synchronization in Chaotic Systems", Physical Review Letters 64, 821, 1990.

[3] L.M. Pecora, "Overview of Chaos and Comunications Research". in "Chaos in Communications", SPIE Proceedings, 2038, 2-25, SPIE. Bellingham, WA. 1993.

[4] T.L. Carroll and L.M. Pecora, "Synchronizing Chaotic Circuits", IEEE Trans. on Circuits and Systems, 38, 453, 1991.

[5] Several examples are given in "Chua's Circuit: A Paradigm for Chaos". Ed.: R.N. Madan. World Scientific Series on Nonlinear Science. World Scientific. London. 1993.

[6] A. González-Marcos and J.A. Martín-Pereda. "Quasi-chaotic digital behavior in an optically processing element". SPIE, 2038, 67-77, 1993.

[7] A. González-Marcos \& J.A. Martín-Pereda. "Sequences of bifurcations and transition to Chaos in an Optically-Processing Element". In Optical Computing, B.S. Wherrett, Ed., 621-624,. Inst. Phys. Conf. Series. No. 139 Part IV. 1995.

[8] J.A. Martín-Pereda \& A. González-Marcos: "Some Connections between Neurophysiology and Optical Computing based on the Theory of Complexity". En "Fluctuation Phenomena: Disorder and Nonlinarity". Eds.: J. Bishop \& L. Vázquez. pp. 107-113. World Scientific Press. Singapur. 1995.

[9] J.A. Martín-Pereda \& A. González-Marcos, "Digital chaos analysis in optical logic structures". SPIE's Photonics East'95 Symposium. Philadelphia, PA. 22-26 Octubre, 1995.

[10] A. González-Marcos \& J.A. Martín-Pereda, "Digital Chaotic Output from an Optically-Processing Element". Optical Engineering. 35, 525-535 (1996).

[11] K. Ikeda, "Multiple-valued stationary state and its instability of the transmitted light by a ring cavity system", Opt. Commun., 30, 257-261 (1979).

[12] M. Okada and K. Takizawa, "Instability of an electrooptic bistable device with a delayed feedback", IEEE J. Quantum Electron., QE-17, 2135-2140 (1981).

[13] A. Neyer and E. Voges, "Dynamics of Electrooptic Bistable Devices with Delayed Feedback", IEEE J. Quontum Electron., QE-18, 2009-2015 (1982).

[14] A. Lempel and J.Ziv, "On the complexity of Finite Sequences". IEEE Transac. Inform. Theory, IT-
22, 75-81 (1976).

[15] R. Singh and D.D. Joseph. "Autoregressive Methods for Chaos on Binary Sequences for the Lorentz Attractor". Phys. Lett. A 135, $247-9$ (1989). 